\title{
On Unresolved Mechanisms of Large Scale Deflagrations in Complex Geometries
}

Makarov, D., Verbecke, F., Keenan, J., \& Molkov, V. (2011). On Unresolved Mechanisms of Large Scale Deflagrations in Complex Geometries. In Unknown Host Publication (pp. 93-103). Research Publishing. https://doi.org/10.3850/978-981-08-7724-8_02-02

Link to publication record in Ulster University Research Portal

\section{Published in:}

Unknown Host Publication

Publication Status:

Published (in print/issue): 01/04/2011

DOI:

10.3850/978-981-08-7724-8_02-02

\section{Document Version}

Publisher's PDF, also known as Version of record

\section{General rights}

Copyright for the publications made accessible via Ulster University's Research Portal is retained by the author(s) and / or other copyright owners and it is a condition of accessing these publications that users recognise and abide by the legal requirements associated with these rights.

\section{Take down policy}

The Research Portal is Ulster University's institutional repository that provides access to Ulster's research outputs. Every effort has been made to ensure that content in the Research Portal does not infringe any person's rights, or applicable UK laws. If you discover content in the Research Portal that you believe breaches copyright or violates any law, please contact pure-support@ulster.ac.uk. 


\title{
On Unresolved Mechanisms of Large Scale Deflagrations in Complex Geometries
}

\author{
Makarov, D.*, Verbecke, F., Keenan, J. and Molkov, V. \\ Hydrogen Safety Engineering and Research Centre (HySAFER), \\ University of Ulster, Newtownabbey, Co. Antrim, BT37 OQB, UK \\ *Corresponding author email:dv.makarov@ulster.ac.uk
}

\begin{abstract}
The paper describes the numerical study performed in order to identify the credible mechanisms of combustion enhancement during hydrogen-air deflagration in a largescale complex geometry of a mock-up hydrogen refuelling station. The Very Large Eddy Simulation (VLES) combustion model, developed at the University of Ulster, and accounting currently for four mechanisms affecting turbulent burning velocity (unburned mixture flow turbulence, turbulence generated by flame front itself, fractal nature of turbulent flame, and preferential diffusion) is applied to simulate the experimental deflagration. Under-prediction of recorded maximum overpressures is attributed to other flame acceleration mechanisms, not yet accounted for in the model. Phenomena capable of contributing to the increase of mass burning rate are suggested including Rayleigh-Taylor instability, increase of the flame front area due to vortexflame interactions, and as yet unidentified behaviour of the fractals sub-model parameters. The simulations of flow acceleration and local pressure dynamics were analysed to scrutinise the assumed mechanisms.
\end{abstract}

KEYWORDS: Hydrogen, deflagration, large-eddy simulation, Rayleigh-Taylor instability, vorticity, fractals.

\section{INTRODUCTION}

The introduction of hydrogen as one of the vectors in energy storage technologies is unavoidable. It is envisaged that the roll-out of hydrogen infrastructure, including refuelling stations, will reach the consumer market in 2015-2020. The construction industry, safety engineers, and health and safety authorities will need validated contemporary tools such as Computational Fluid Dynamics (CFD) to predict hazards and associated risks of realistic hydrogen accident scenarios, including hydrogen-air deflagration.

To be a reliable predictive tool CFD models have to be built on validated sub-models describing various physical phenomena occurring at different stages of deflagration development, scales, concentrations, in arbitrary geometries and with realistic level of congestion. It is widely accepted that the Large Eddy Simulation (LES) provides a better description of turbulent flows at the resolved level, especially of large scale unsteady motions [1]. This paper describes the numerical study performed in order to identify and quantify the credible mechanisms of combustion enhancement during hydrogen-air deflagration in a large-scale geometry of a hydrogen refuelling station [2], using the Very Large Eddy Simulation (VLES) deflagration model developed at the University of Ulster during the last decade.

Proc. of the Sixth International Seminar on Fire \& Explosion Hazards (FEH6)

Edited by D. Bradley, G. Makhviladze and V. Molkov

Copyright (c) 2011 CPD Unit, University of Leeds :: Published by Research Publishing

ISBN: 978-981-08-7724-8 :: doi:10.3850/978-981-08-7724-8_02-02

www.rpsonline.com.sg 


\section{DETAILS OF DEFLAGRATION EXPERIMENT}

Shell Global Solutions (UK) and the Health and Safety Laboratory (UK) conducted a series of hydrogen deflagration experiments in a geometry representing a realistic retail hydrogen refuelling station [2]. An experiment with deflagration of uniform near stoichiometric hydrogen-air cloud, designed to represent the worst-case scenario, was chosen to develop further the VLES deflagration model.

The experimental setup and its dimensions are shown in Fig. 1. The setup comprised a dummy car, two dispenser units $(2.1 \mathrm{~m}$ high) and a confining wall. The car passenger section was welded to prevent hydrogen penetration inside, but the engine bay $(1.7 \mathrm{~m}$ wide, 0.8 high, $0.7 \mathrm{~m}$ long) was open from the bottom to allow to be filled with flammable mixture. The hydrogen-air mixture was prepared and retained under a plastic film held by a metal frame (5.4 m wide, $6.0 \mathrm{~m}$ long and $2.5 \mathrm{~m}$ high), fixed to the confining wall. The total volume of the hydrogen-air mixture, excluding sealed dispensers and the passenger section of the dummy car, was $70.16 \mathrm{~m}^{3}$ (approximate blockage ratio is $13.6 \%$ ). The hydrogen concentration in the mixture was $31.4 \%$ (vol.), mixture temperature was equal $28.9^{\circ} \mathrm{C}$, and relative humidity $-42.1 \%$. The igniter was located between the dispensers, at a height of $1.25 \mathrm{~m}$ and at a distance of $1.3 \mathrm{~m}$ from the confining wall. Location of the pressure transducers is shown in Fig. 2.

\section{DEFLAGRATION MODEL}

Numerical simulations were conducted using the deflagration model accounting for four major mechanisms contributing to the augmentation of turbulent burning velocity: flow turbulence, turbulence generated by the flame front itself, fractal-like flame front wrinkling, and preferential diffusion or the leading point mechanism. The latest version of the VLES deflagration model is described elsewhere [3, 4] and only its main features are given below.
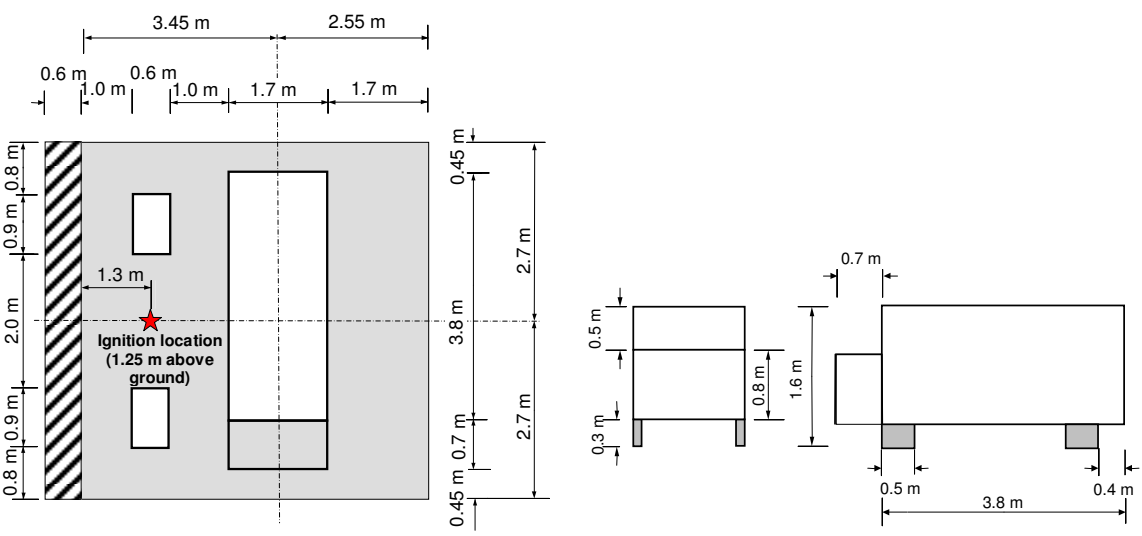

Figure 1. Layout and dimensions of the experimental setup and mock-up car. 


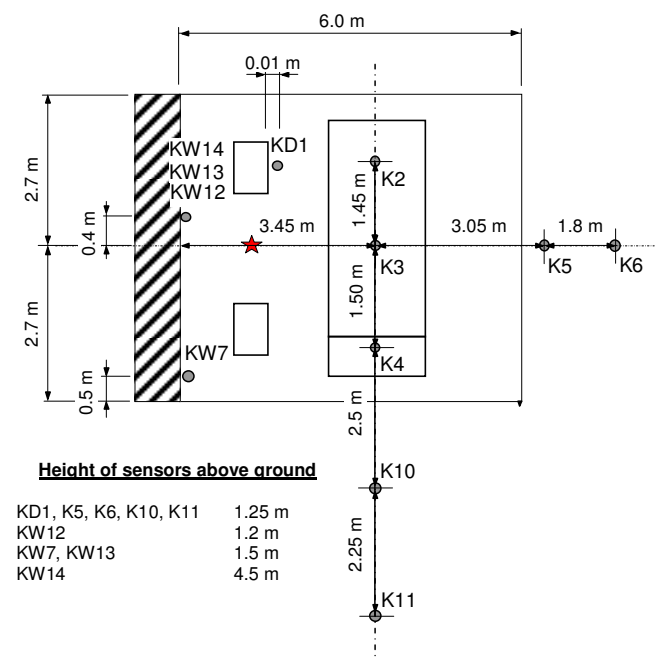

Figure 2. Location of pressure transducers.

The resolution of all scales controlling mixing and chemical reaction processes is not possible with present computer power, and all Sub-Grid Scale (SGS) processes have to be modelled. Following Pope [1] we call the described below model as Very Large Eddy Simulation (VLES) deflagration model.

The model comprises three-dimensional filtered equations of mass, momentum, energy and species conservation. The premixed flame propagation is modelled using the progress variable equation

$$
\frac{\partial}{\partial t}(\bar{\rho} \tilde{c})+\frac{\partial}{\partial x_{j}}\left(\bar{\rho} \tilde{u}_{j} \tilde{c}\right)=\frac{\partial}{\partial x_{j}}\left(\frac{\mu_{e f f}}{S c_{e f f}} \frac{\partial \tilde{c}}{\partial x_{j}}\right)+\bar{S}_{c},
$$

where the gradient method is used to express the source term in a form $\overline{S_{c}}=\rho_{u} S_{t}|\operatorname{grad} \tilde{c}|$ to calculate the mass burning rate.

The effect of flow turbulence on burning velocity is modelled using Yakhot's model [5]. Turbulence generated by the flame front itself is accounted for based on the work by Karlovitz et al. [6]. Fractal theory is used to describe the fractal increase of turbulent flame front area when the flame radius $R$ is equal, or above, the critical radius $R_{0}$ for transition to a self-similar regime. The empirical dependence by North and Santavicca [7] was used for the fractal dimension. The inner cut-off scale, $\lambda$, is assumed to be proportional to the laminar flame thickness. The effect of the preferential diffusion on the burning velocity is calculated according to Zimont and Lipatnikov [8].

Eventually, the model expression for the turbulent SGS burning velocity during transition from laminar to the fully developed turbulent combustion regime $\left(0<R<R_{0}\right)$ is 
$S_{t}=\left[S_{u}\left(Y_{H_{2}}, T, p\right) \cdot \chi_{l p} \cdot\left\{1+\left(\psi \cdot \chi_{\text {Kar }_{\text {MuX }}}-1\right) \cdot\left[1-\exp \left(-\frac{R}{R_{0}}\right)\right]\right\}\right] \cdot \exp \left(\frac{u^{\prime}}{S_{t}}\right)^{2}$,

and for $R>R_{0}$

$S_{t}=\left[S_{u}\left(Y_{H_{2}}, T, p\right) \cdot \chi_{t p} \cdot\left\{1+\left(\psi \cdot \chi_{\text {Kar }_{\text {uax }}}-1\right) \cdot\left[1-\exp \left(-\frac{R}{R_{0}}\right)\right]\right\} \cdot\left(\frac{\lambda_{R_{0}}}{\lambda} \cdot \frac{R}{R_{0}}\right)^{D-2}\right] \cdot \exp \left(\frac{u^{\prime}}{S_{t}}\right)^{2}$,

where $S_{t}$ is turbulent burning velocity, $S_{u}\left(Y_{H_{2}}, T, p\right)$ laminar burning velocity as a function of temperature, pressure and hydrogen concentration, $\chi_{l p}$ flame wrinkling factor due to the leading point mechanism, $\psi$ empirical coefficient, $\chi_{\text {Karl } l_{M A X}}$ maximum flame wrinkling factor due to turbulence generated by the flame front itself, $R$ flame radius/distance to ignition point, $R_{0}$ critical radius for transition to the selfsimilar regime of turbulent flame propagation, $\lambda$ inner cut-off scale, $\lambda_{R_{0}}$ inner cut-off scale at the flame critical radius $R_{0}, u^{\prime}$ SGS velocity, and $D$ fractal dimension.

The only model calibration coefficient, $\psi$, was found to vary within the limits $\psi=0.4-0.5$. The model was successfully validated against 21 large-scale hydrogenair deflagration experiments, including closed vessels, vented deflagrations, open atmosphere deflagrations, deflagrations in a tunnel, and non-uniform mixture deflagrations (see [4]).

The described VLES model was realised using FLUENT software. The calculation domain 20x20x10 m was meshed using an unstructured tetrahedral grid. The characteristic Control Volume (CV) size was $0.0375 \mathrm{~m}$ under the car, 0.15-0.20 m within the refuelling station and up to $2.0 \mathrm{~m}$ in the far field. The total number of CVs was $1,184,228$. A coupled compressible solver with an explicit scheme for time integration was applied with a $C F L$ number equal to 0.8 . The second-order upwind difference scheme was applied for discretisation of convection terms, the second-order central scheme for diffusion terms, and four stage Runge-Kutta scheme for time stepping.

\section{ANALYSIS OF POTENTIAL MECHANISMS}

The straightforward application of the above combustion model with the value of the empirical coefficient $\psi=0.4$ results in under-prediction of the maximum explosion overpressure. To make the simulated pressure transients closer to those of experiment an additional flame wrinkling factor with a maximum value of 2.5 was applied in a space between the ground and the car bottom and within the engine compartment. This was achieved by adjusting the value of the empirical coefficient for turbulence generated by flame front itself from $\psi=0.4$ to maximum $\psi=1$ during $10 \mathrm{~ms}$, starting from the moment when a leading flame edge entered the space between the car and the ground. Comparison of both simulation results with the experimental data for pressure gauges K3, K5, and K10 is given in Fig. 3. 
It is worth noting that a similar additional flame wrinkling factor of the order of 2 was identified previously to reproduce experimental pressure dynamics in VLES simulations of a vented methane-air deflagration in the large-scale SOLVEX facility [9]. It was shown that to match experimental coherent deflagrations pressure dynamics the factor had to be applied only to a gradual increase of the turbulent burning velocity in the area of the external explosion. Another example of underresolved VLES of the coherent deflagrations with relatively large vent opening is described in [10].

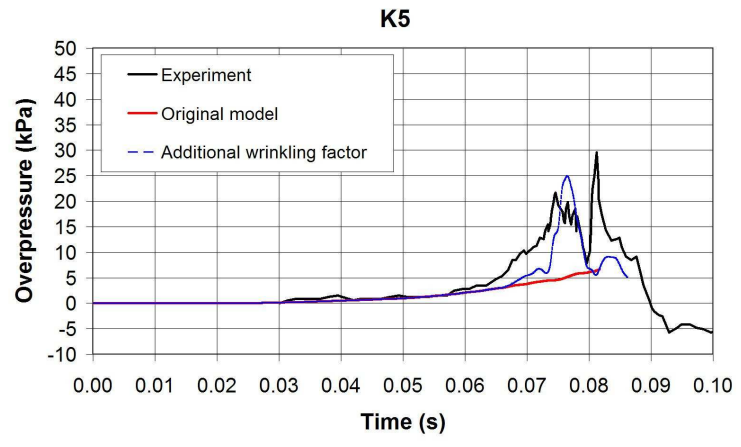

a)

K3
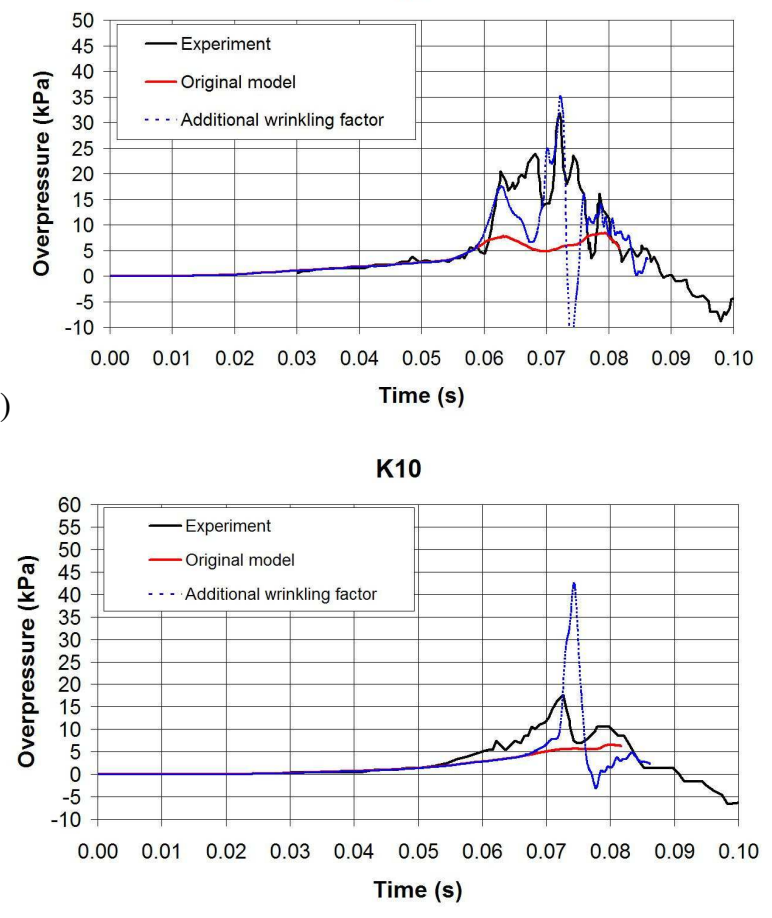

Figure 3. Experimental and simulated pressure dynamics. 
It is assumed that unidentified mechanism(s) of flame acceleration are not currently accounted in the VLES model, and thus are not contributing to an increase in the turbulent burning velocity and pressure build-up. Three potential mechanisms are considered as contributors, being responsible separately, or in combination, for the unresolved increase of the turbulent burning velocity: Rayleigh-Taylor instability [11], increase of flame front area due to anisotropic vortex-flame interaction, and varying inner cut-off in the fractals sub-model.

\section{Rayleigh-Taylor Instability}

Rayleigh-Taylor instability is the instability of the interface between two fluids of different densities when subject to acceleration from the lighter to the heavier. Rayleigh-Taylor flame instability is known to contribute to deflagration dynamics in an obstructed environment when the flame front accelerates between obstacles. Tsuruda and Hirano [12] highlighted that the Rayleigh-Taylor mechanism appears earliest and develops the most rapid, and was supposed to be a dominating mechanism in flame turbulisation when a flame is passing over an obstacle.

To inspect a potential for initiation and growth of flame front area due to RayleighTaylor instability, the projection of the flow acceleration vector on the direction normal to the flame front (which corresponds to the gradient of the progress variable in the VLES model applied) was numerically visualised using the scalar vector product

$$
f(\vec{a}, \operatorname{grad}(\tilde{c}))=\vec{a} \cdot \frac{\operatorname{grad}(\tilde{c})}{|\operatorname{grad}(\tilde{c})|} .
$$

Figure 4 shows distribution of a function $f(\vec{a}, \operatorname{grad}(\tilde{c}))$ in the central cross section of the calculation domain overlapped with flow acceleration vectors and iso-profiles of the progress variable $c=0.0001$ and $c=0.99$ to indicate the location of the simulated flame front. The maximum value of the function is located on the leading edge of the flame front normal to the direction of the flow acceleration. Analysis shows that values of the function at the leading edge of the flame front in the open space are in the range 2000-2500 s ${ }^{-2}$. However, 4-5 times larger values are under the car and when the flame front exits the space under the car.

Timing of the snapshots shown in Fig. $4 \mathrm{~b}$ and Fig. $4 \mathrm{c}$ roughly corresponds to the timing of the simulated pressure peaks of the original model in sensor K3, located under the car (see Fig. 3). It would be logical to assume that the first experimental pressure peak corresponds to the flame acceleration under the car and the second pressure peak corresponds to the flame acceleration coming from under the car. The simulation with the additional factor seems to reproduce both peaks, in spite of the fact that the additional flame wrinkling factor is applied only under the car and does not account for the flame acceleration on the exit that position. This may be explained by the close location of the modelled flame acceleration area to that in the experiment, such that it makes the simulated and the experimental pressure dynamics in sensor K3 visually similar. 
a)

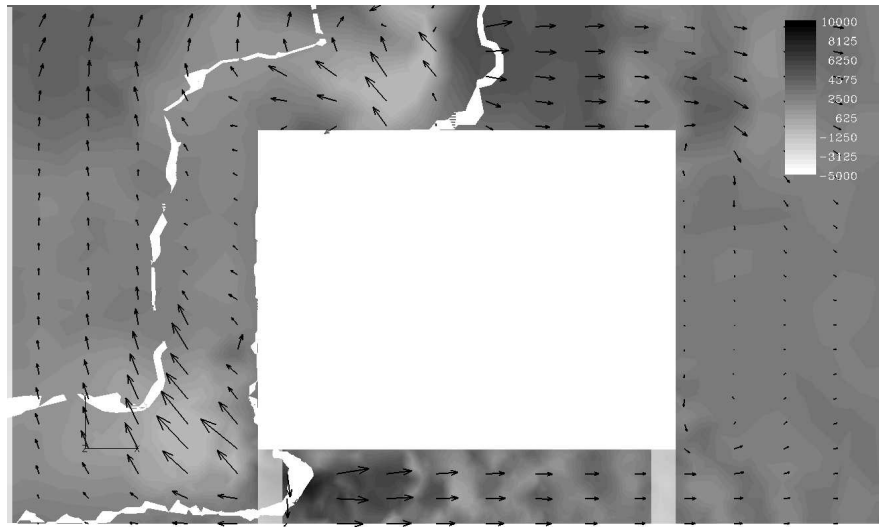

b)

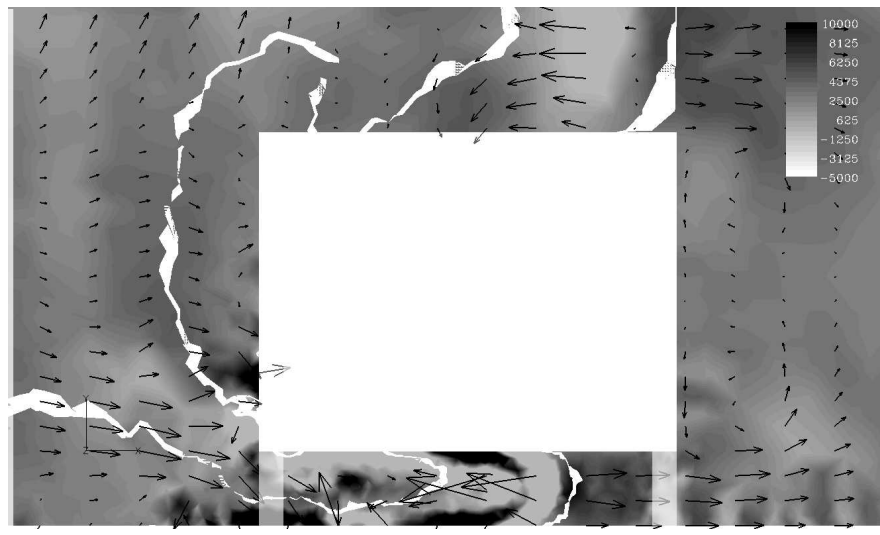

c)

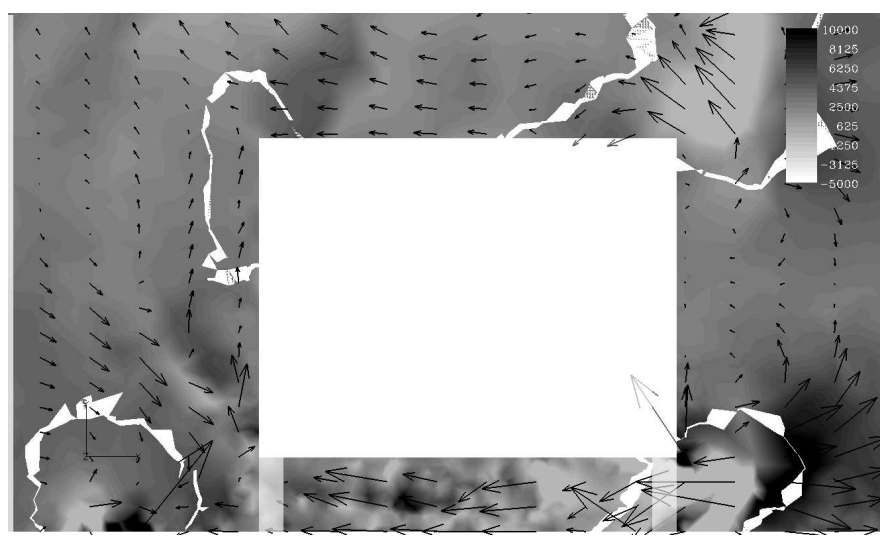

Figure 4. Distribution of the flow acceleration normal to the flame front, (a) $t=5.57 \cdot 10^{-2} \mathrm{~s}$, (b) $t=6.63 \cdot 10^{-2} \mathrm{~s}$, (c) $t=7.31 \cdot 10^{-2} \mathrm{~s}$.

This explanation is supported by an analysis of pressure transients in the location of sensors K5 and K10. The pressure transducer K5 is located closer to the area of flame 
acceleration on the exit from under the car, so that the second experimental pressure peak, recorded by K5, is larger than the first. The modified VLES model, on the contrary, does not have any flame acceleration factors outside of space between the car and the ground; hence the second peak is under-predicted and is smaller than the first one. The first pressure peak obtained with the pressure transducer K10 using the modified VLES model is too high compared to the experimental one, which suggests that the additional flame wrinkling factor of 2.5 applied under the car in the engine bay space is overestimated and the ad-hoc model modification is too simple. Similar to the pressure dynamics in the sensor K5, the second simulated pressure peak in sensor K10 is also under-predicted, which again supports the hypothesis that the flame acceleration due to Rayleigh-Taylor instability takes place on the exit from under the car and must be accounted for in the model.

\section{Anisotropic Vortex-flame Interaction}

The majority of combustion models originate from the assumption of isotropic turbulence and can hardly be expected to reproduce combustion enhancement due to anisotropy of the turbulence in the vorticity field. The LES technique may be a remedy, but not when the filter width is larger than the anisotropic turbulence length scales, as occurs in VLES. This point of view is supported by a recent review [13]: “...standard r.m.s. velocity $u$ ' may not be a proper characteristic of turbulence in a premixed flame. ...An attractive approach to characterising turbulence in premixed flames would be based on analysing vorticity, rather than $u$ '." Though the same review stated that there is little knowledge of vorticity behaviour in premixed flames, the vorticity field around and under the car was analysed to study its potential impact on combustion acceleration. Distribution of the magnitude of vorticity on the resolved scales is shown in Fig. 5. The largest vorticity is observed in the boundary layers close to obstacle surfaces and the areas where the gas flow changes direction. One can see that there is no substantial growth of the vorticity during flame propagation under the car. The vorticity in the flame front even decreases on the flame exits from under the car. The latter makes it questionable to expect any additional growth of the turbulent burning velocity as a function of anisotropic combustion.

\section{Fractals Inner Cut-off and SGS Mixing Length}

Fractal theory is a convenient tool to model the growth of highly contorted surfaces, including turbulent flames, when numerical resolution is insufficient. The application of the fractal theory requires specification of inner and outer cut-off scales. If the outer cut-off scale is uniquely determined by the scale of the flame, definition of the inner cut-off scale is less certain. Currently the flame front thickness is used as the inner cut-off in the VLES model following [14]. Various approaches for definition of the inner cut-off scale, see review in [15], suggest a dependence also on the r.m.s. velocity $u^{\prime}$ and the integral turbulence length scale $\Lambda$. However, unambiguous calculation of these field parameters is inherently complicated by the resolution of the VLES simulations. For example, if one tries to estimate an inner cut-off as the turbulent mixing length based on the VLES field parameters, a value proportional to the VLES filter width would be obtained, even using RNG SGS model, where the effective viscosity dependence on a filter width is not linear. More ideas and efforts are needed in this direction of VLES model development. 
Proc. of the Sixth International Seminar on Fire \& Explosion Hazards (FEH6) 101

a)

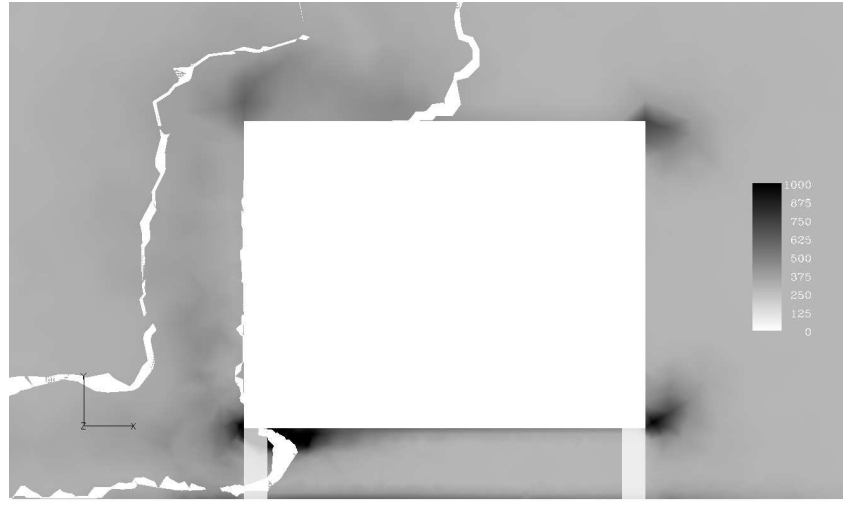

b)

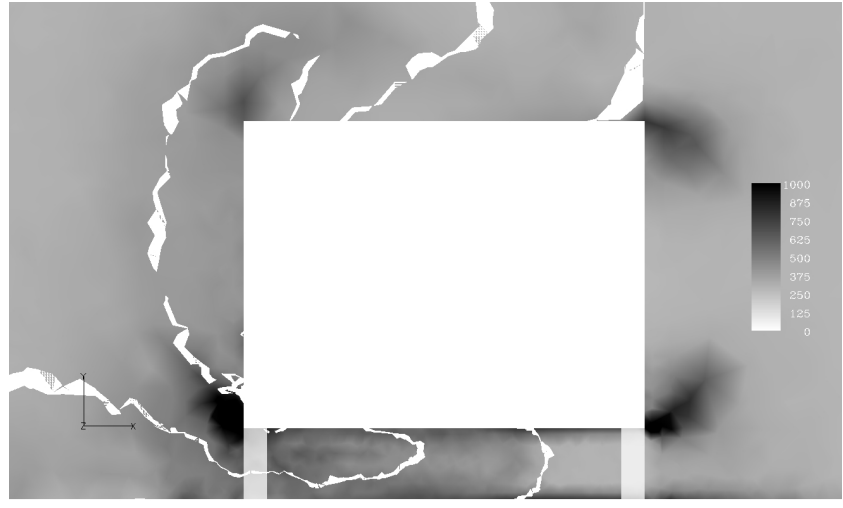

c)

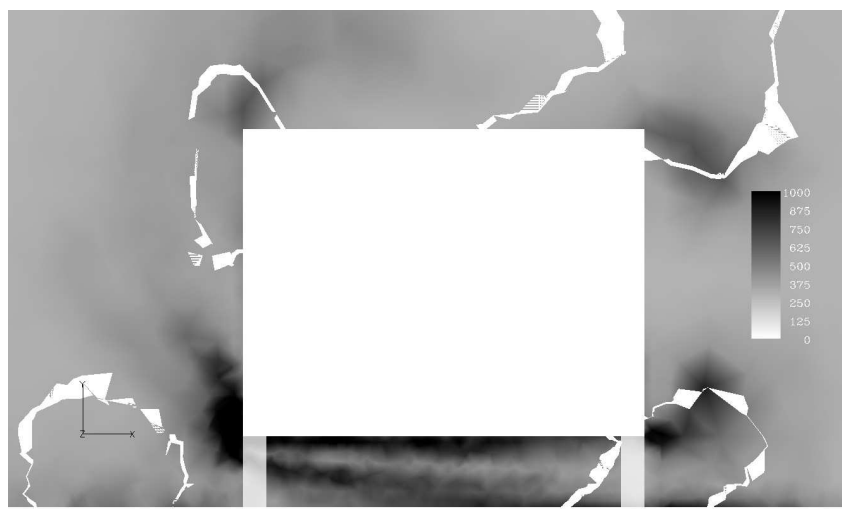

Figure 5. Distribution of vorticity in the central cross section, (a) $t=5.57 \cdot 10^{-2} \mathrm{~s}$, (b) $\left.t=6.63 \cdot 10^{-2} \mathrm{~s}, \mathrm{c}\right) t=7.31 \cdot 10^{-2} \mathrm{~s}$. 


\section{CONCLUSIONS}

A numerical analysis of the large scale hydrogen-air deflagration experiment in a mock-up refuelling station has been undertaken. Three physical mechanisms, capable potentially of contributing to flame wrinkling and pressure build-up, but not accounted for in the current VLES deflagration model, were scrutinised: RayleighTaylor instability, potential increase of the flame front area due to anisotropic vortexflame interactions, and varying inner cut-off in the fractals sub-model.

The Rayleigh-Taylor instability was identified as the most likely missing contribution to the combustion enhancement in the VLES model compared to the experiment. The areas of flow acceleration in the direction of Rayleigh-Taylor instability were identified as being under the car and where the flame exits the space under the car similar to [12].

The effect of vorticity on the increase in flame front area through the anisotropic flame-vortex interactions also should be studied further. It seems unlikely that vorticity will contribute to the same extent as Rayleigh-Taylor instability to the varying turbulent burning velocity.

More ideas and research are needed for better use of conclusions of the fractals theory in modelling of flame surface area at unresolved scales.

\section{REFERENCES}

1. Pope, S.B., Turbulent flows, Cambridge University Press, 2000.

2. Shirvill, L.C., Royle, M., and Roberts, T.A., "Hydrogen Releases Ignited in a Simulated Vehicle Refuelling Environment", Proc. of 2nd Int. Conference for Hydrogen Safety, San Sebastian, Spain, 11-13 September 2007.

3. Verbecke, F., Makarov, D., and Molkov, V., "VLES of Lean Hydrogen-Air Deflagrations in a Closed 5.7 m Height Vessel", Proc. of 6th Mediterranean Combustion Symposium, Ajaccio, France, 7-11 June 2009.

4. Verbecke, F., Formation and Combustion of Non-Uniform Hydrogen-Air Mixtures, $\mathrm{PhD}$ thesis, University of Ulster, 2009.

5. Yakhot, V., "Propagation Velocity of Premixed Turbulent Flames", Comb. Sci. Tech. 60: 191-214 (1988).

6. Karlovitz, B., Denniston, D.W.Jr., and Wells, F.E., "Investigation of Turbulent Flames", The Journal of Chemical Physics 19: 541-547 (1951).

7. North, G.L., and Santavicca, D.A., “The Fractal nature of Premixed Turbulent Flames”, Comb. Sci. Tech. 72: 215-232 (1990).

8. Zimont, V.L., and Lipatnikov, A.N., "A Numerical Model of Premixed Turbulent Combustion of Gases”, Chem. Phys. Reports 14: 993-1025 (1995).

9. Molkov, V., Makarov, D., and Puttock, J., "The Nature and Large Eddy Simulation of Coherent Deflagrations in a Vented Enclosure-atmosphere System", J. Loss Prev. Proc. Industries 19: 121-129 (2006). 
10. Bauwens, C.R., Chaffee, J., and Dorofeev, S., "Experimental and Numerical Study of Methane-Air Deflagrations in a Vented Enclosure", Proc. of 9th Int. Symp. on Fire Safety Science, Karlsruhe, Germany, 21-26 September 2008.

11. Dorofeev, S., FM Global, USA, Personnal communication, 2009.

12. Tsuruda, T., and Hirano, T., "Growth of Flame-front Turbulence during Flame Propagation across Obstacles”, Comb. Sci. Tech. 51: 323-328 (1987).

13. Lipatnikov, A., and Chomiak, J., "Effects of Premixed Flames on Turbulence and Turbulent Scalar Transport”, Prog. Energy Comb. Sci. 36: 1-102 (2010).

14. Gülder, O.L., "Turbulent Premixed Combustion Modelling using Fractal Geometry", Proc. Comb. Inst. 23: 835-842 (1990).

15. Gülder, O., and Smallwood, G., "Inner Cut-off Scale of Flame Surface Wrinkling in Turbulent Premixed Flames”, Comb. Flame 103: 107-114 (1995). 